\title{
Recent results from PHENIX at RHIC
}

\author{
Victor Riabov ${ }^{1,2, *}$ \\ ${ }^{1}$ PNPI of NRC "Kurchatov Institute", Orlova rostcha-1, Gatchina, 188300, Russia \\ ${ }^{2}$ National Research Nuclear University MEPhI (Moscow Engineering Physics Institute), Kashirskoe \\ highway 31, Moscow, 115409, Russia
}

\begin{abstract}
The PHENIX experiment at the relativistic heavy ion collider (RHIC) finished data taking in 2016. However, large datasets collected in different collision systems $(\mathrm{p}+\mathrm{p}, \mathrm{p}+\mathrm{A}$ and $\mathrm{A}+\mathrm{A})$ at different energies $\left(\sqrt{ }_{\mathrm{NN}}=19-500 \mathrm{GeV}\right)$ during the last years of the detector operation are actively analysed by the collaboration and bring a wealth of new experimental results. This paper reviews the most recent PHENIX results on the light flavour hadron production, yields and angular correlations of the direct photons in heavy-ion collisions as well as on the search for the onset of collectivity in high multiplicity $\mathrm{p}+\mathrm{p}$ and $\mathrm{p}+\mathrm{A}$ collisions.
\end{abstract}

\section{Introduction}

The scientific mission of the PHENIX experiment is a detailed study of properties of the strongly interacting quark-gluon plasma (QGP) produced in central heavy-ion collisions at RHIC [1]. The properties of the QGP are studied by measuring differential yields and angular distributions of different particles produced in the final state. Since particles are produced throughout the system evolution and their properties are affected by the late hadronic phase, it is vital to measure as many signatures of the QGP as possible in dependence on a collision system size and collision energy. In the last years, a great attention was also paid to the search for the onset of collectivity in high-multiplicity collisions of small systems.

In this paper, we present the most recent PHENIX results for the direct photon production in $\mathrm{p}+\mathrm{Au}, \mathrm{Cu}+\mathrm{Cu}$ collisions at $\sqrt{\mathrm{s}_{\mathrm{NN}}}=200 \mathrm{GeV}$ and in $\mathrm{Au}+\mathrm{Au}$ collisions at $\sqrt{\mathrm{s}_{\mathrm{NN}}}=39$ and $62.4 \mathrm{GeV}$, measurement of flow coefficients in $\mathrm{p} / \mathrm{d} /{ }^{3} \mathrm{He}+\mathrm{Au}$ collisions in a wide range of energies $\sqrt{\mathrm{s}_{\mathrm{NN}}}=19.6-200 \mathrm{GeV}$.

\section{Direct photon production}

PHENIX invested many efforts in the measurement of low transverse momentum $\left(\mathrm{p}_{\mathrm{T}}\right)$ direct photons in different collision systems. By direct photons we mean all photons in the event

* e-mail: riabov vg@pnpi.nrcki.ru 
that do not originate from hadron decays. Such direct photons are produced throughout the system evolution. The interest to low- $\mathrm{p}_{\mathrm{T}}$ direct photons is motivated by expectations that in a limited $\mathrm{p}_{\mathrm{T}}$ range, from $\sim 1 \mathrm{GeV} / \mathrm{c}$ to $\sim 4 \mathrm{GeV} / \mathrm{c}$, the production of such photons in heavyion collisions is dominated by radiation from the QGP.

Several years ago, PHENIX reported results on the direct photon production and direct photon flow in $\mathrm{Au}+\mathrm{Au}$ collisions at $\sqrt{\mathrm{s}_{\mathrm{NN}}}=200 \mathrm{GeV}$. A significant excess of the direct photon yields over the binary-scaled results in $\mathrm{p}+\mathrm{p}$ was observed in central collisions at $\mathrm{p}_{\mathrm{T}}$ $<4 \mathrm{GeV} / \mathrm{c}$ [2]. The observed excess was attributed to thermal radiation. At the same time the flow coefficients $v_{2}$ and $v_{3}$ measured for direct photons turned out to be comparable to those measured for charged hadrons [3]. The theoretical models cannot simultaneously describe the yield and the flow measurements for direct photons. The large yields of direct photons suggest that the photons are radiated very early in the system evolution while the interacting system is still very hot. However, the large flow of direct photons suggests that they are radiated late in the system evolution when the system has enough time to develop the collective flow. Similar situation with the yield and flow measurements for low- $\mathrm{p}_{\mathrm{T}}$ direct photons was later observed at the LHC at ten times higher energy of ${\sqrt{\mathrm{s}_{\mathrm{NN}}}}=2760$ $\mathrm{GeV}$ [4]. This situation calls for a systematic study of the direct photon production as a function of a collision system size and energy.

Recently, PHENIX measured the direct photon production in $0-5 \%$ most central $\mathrm{p}+\mathrm{Au}$ collisions at $\sqrt{ }_{\mathrm{N}_{\mathrm{N}}}=200 \mathrm{GeV}$. The results of the measurements are presented in Fig. 1. The smooth curve with a band shows the $\mathrm{p}+\mathrm{p}$ reference scaled by the number of binary collisions $\mathrm{N}_{\text {coll }}$. A clear enhancement of the direct photon production over the binary-scaled $p+p$ reference is observed. This is the first observation of the excessive direct photon production in collisions of small systems at RHIC.

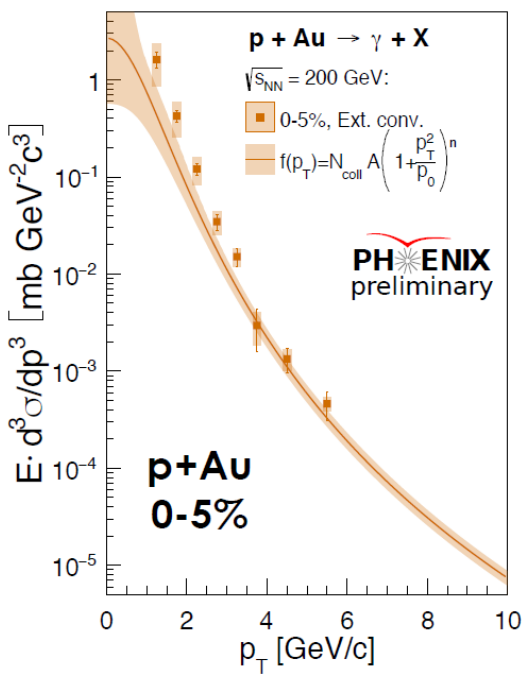

Figure 1. Invariant differential yield of direct photons in $0-5 \%$ most central $\mathrm{p}+\mathrm{Au}$ collisions at $\sqrt{ } \mathrm{s}_{\mathrm{NN}}=200 \mathrm{GeV}$. The smooth curve and the shaded region correspond to the binary-scaled $\mathrm{p}+\mathrm{p}$ reference 
Fig. 2 shows the PHENIX results for the direct photon production in $\mathrm{Cu}+\mathrm{Cu}$ collisions at $\sqrt{\mathrm{s}_{\mathrm{NN}}}=200 \mathrm{GeV}$ [5] and in $\mathrm{Au}+\mathrm{Au}$ collisions at $\sqrt{ }_{\mathrm{S}_{\mathrm{NN}}}=39$ and $62.4 \mathrm{GeV}$ [6]. The copper nucleus is smaller than the gold one and hence measurements in $\mathrm{Cu}+\mathrm{Cu}$ collisions can improve understanding of the direct photon production in a region of relatively small values of number of participants $\mathrm{N}_{\text {part }}$, central $\mathrm{Cu}+\mathrm{Cu}$ collisions correspond to semi-central $\mathrm{Au}+\mathrm{Au}$ collisions by $\mathrm{N}_{\text {part }}$. The direct photon yields were measured in minimum bias and 0$40 \%$ most central $\mathrm{Cu}+\mathrm{Cu}$ collisions. Similar to the case of $\mathrm{Au}+\mathrm{Au}$ collisions, a significant excess of the direct photon production over the expectations based on the measurements in $\mathrm{p}+\mathrm{p}$ is observed. In this case, the $\mathrm{p}_{\mathrm{T}}$ spectra and integrated yields measured in $\mathrm{Cu}+\mathrm{Cu}$ are found to be in a very good agreement with $\mathrm{Au}+\mathrm{Au}$ results at a similar number of participants. Measurements at lower energies can be used to study the direct photon production as a function of collision energy. As in previous cases, a substantial excess of the direct photon yields at low momentum $\mathrm{p}_{\mathrm{T}}<3 \mathrm{GeV} / \mathrm{c}$ is observed. The excess is observed with respect to binary-scaled pQCD calculations in $\mathrm{p}+\mathrm{p}$ collisions at corresponding energies due to lack of the measured $p+p$ references.

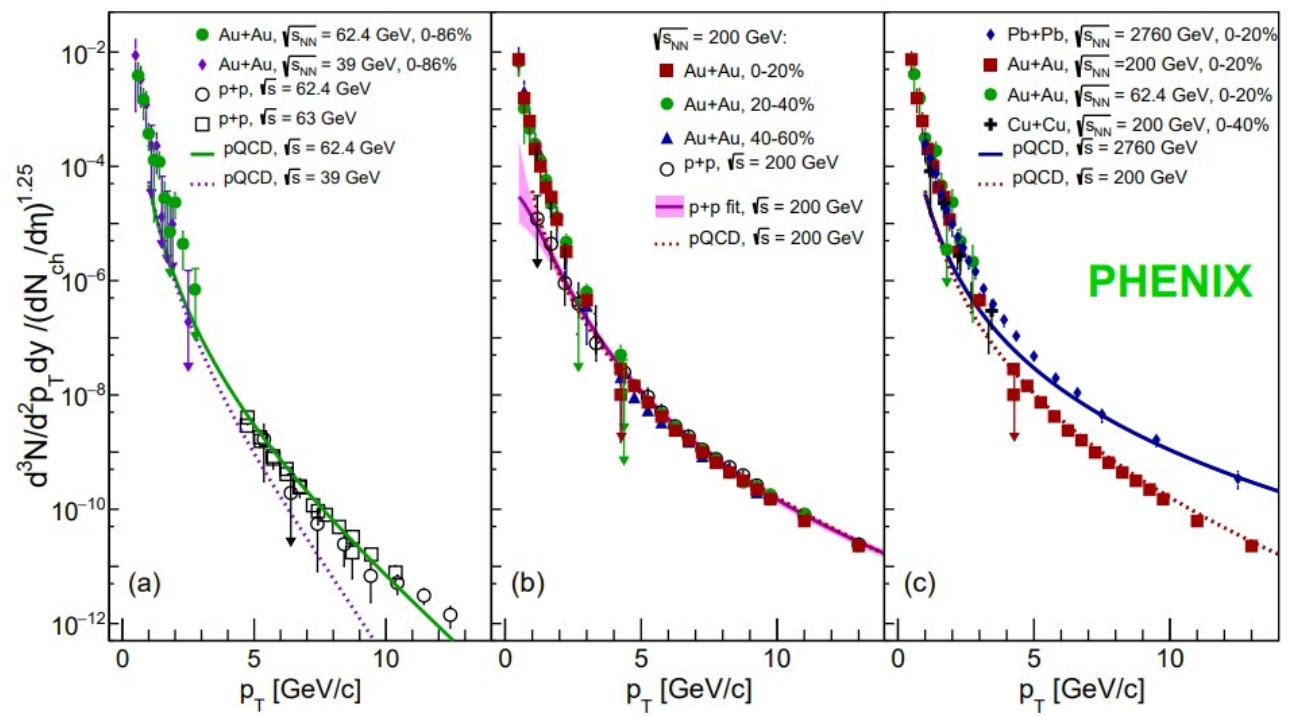

Figure 2. Differential yields of the direct photons measured in different collision systems as a function of transverse momentum. The yields are divided by the changed particle multiplicity density to the power of 1.25 to reveal the scaling discussed in the text

Fig. 2 demonstrates a very interesting scaling for the direct photon production in heavyion collisions at RHIC and the LHC [2-6]. When the photon yields are scaled by $\left(\mathrm{dN}_{\mathrm{ch}} /\right.$ $\mathrm{d} \eta)^{1.25}$, the whole variety of measurements splits in two different domains. At high transverse momenta $4 \mathrm{GeV} / \mathrm{c}<\mathrm{p}_{\mathrm{T}}<20 \mathrm{GeV} / \mathrm{c}$, the production of direct photons is separated by a collision energy. What is surprising is that at low momenta $\mathrm{p}_{\mathrm{T}}<3-4 \mathrm{GeV} / \mathrm{c}$, all yields measured at different centralities and different collision energies in the range of $\sqrt{ }_{\mathrm{S}_{\mathrm{NN}}}=39-2760 \mathrm{GeV}$ scale to the same universal curve. 
Fig. 3 demonstrates the same scaling but presented in a different way. It shows the direct photon yields integrated at $\mathrm{p}_{\mathrm{T}}>1 \mathrm{GeV} / \mathrm{c}$, in the momentum range where the direct photon production in heavy-ion collisions is dominated by the thermal radiation. The integrated yields are shown as a function of the charged particle multiplicity. Points of different colours correspond to $\mathrm{Cu}+\mathrm{Cu}, \mathrm{Au}+\mathrm{Au}$ and $\mathrm{PbPb}$ collisions at different energies, $\sqrt{\mathrm{s}_{\mathrm{NN}}}=39-2760 \mathrm{GeV}$. All measurements in heavy-ion collisions follow the same line having a slope of 1.25. It also means that the yields of direct photons grow faster than the average charged particle multiplicity. The results in $\mathrm{p}+\mathrm{p}$ collisions are represented in this figure with $\mathrm{p}_{\mathrm{T}}$-integrated $\mathrm{pQCD}$ curves. Again, the yields show the universal scaling with multiplicity with the same slope value of 1.25. Points corresponding to small collision systems, these are $\mathrm{p}+\mathrm{Au}$ and $\mathrm{d}+\mathrm{Au}$ [7] collisions at ${\sqrt{\mathrm{s}_{\mathrm{NN}}}}=200 \mathrm{GeV}$, fall out of this scaling and demonstrate a different behaviour suggesting the possible turn on of thermal radiation from $\mathrm{p}+\mathrm{p}$ to heavy-ion collisions. There is no unique explanation of the observed scaling now. However, it may indicate the large direct photon production near the phase transition to the hadronic phase.

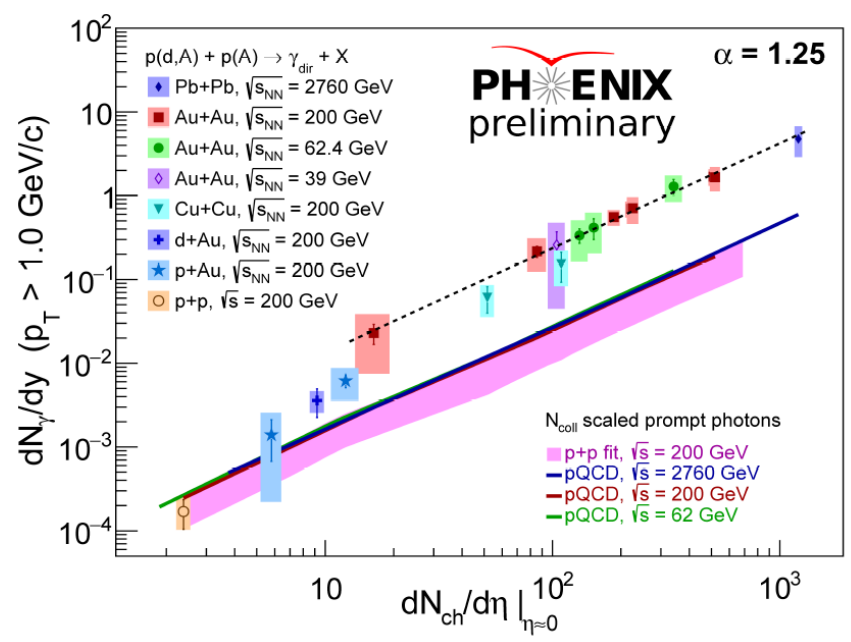

Figure 3. Direct photon yields integrated at $\mathrm{p}_{\mathrm{T}}>1 \mathrm{GeV} / \mathrm{c}$ shown as a function of charged particle multiplicity at midrapidity $\mathrm{dN}_{\mathrm{ch}} /\left.\mathrm{d} \eta\right|_{\eta=0}$

\section{Collective flow in small systems}

Historically, the observation of the large particle flow in heavy-ion collisions at RHIC and LHC energies has been interpreted to indicate the formation of a strongly coupled QGP with properties of a nearly perfect fluid [1]. Later, similar flow signatures were observed in high-multiplicity collisions of small systems [8,9]. The interpretation of these results is still incomplete and this situation calls for a systematic study of various observables, including the flow, in different small collision systems. As a response to these needs, PHENIX performed two experimental programs. The geometry scan program included the study of $\mathrm{p}+\mathrm{Au}, \mathrm{d}+\mathrm{Au}$ and ${ }^{3} \mathrm{He}+\mathrm{Au}$ collisions at the same energy of $\sqrt{ }_{\mathrm{NN}}=200 \mathrm{GeV}$. This program was intended to study a relation between the initial geometry of the nuclear overlap region 
and the final state particle momentum anisotropies. The energy scan program included the study of $\mathrm{d}+\mathrm{Au}$ collisions at four different energies of $\sqrt{ }_{\mathrm{S}_{\mathrm{NN}}}=200,62.4,39$ and $19.6 \mathrm{GeV}$. This program aimed at studying the relation between the initial temperature and lifetime of the medium and the collectivity signatures.

\subsection{Geometry scan program}

Over the years, RHIC delivered collisions of $\mathrm{p} / \mathrm{d} /{ }^{3} \mathrm{He}$ with $\mathrm{Au}$ at $\sqrt{\mathrm{s}_{\mathrm{NN}}}=200 \mathrm{GeV}$. It is obvious that three systems have very different initial geometries. In order to quantify these geometries, we calculated the average second and third order spatial eccentricities for such collisions using the Glauber Monte-Carlo model [10]. The results of these calculations are shown in the right panel of Fig. 4. It turned out that $\mathrm{d}+\mathrm{Au}$ and ${ }^{3} \mathrm{He}+\mathrm{Au}$ collisions have very similar second order eccentricities, which are significantly larger than those in $\mathrm{p}+\mathrm{Au}$ collisions. The third order eccentricity has a very clear hierarchy and it is the largest in ${ }^{3} \mathrm{He}+\mathrm{Au}$ collisions. It is clear why; the initial state has an intrinsic triangularity from three nucleons in the ${ }^{3} \mathrm{He}$ nucleus. Left panel of the same figure shows the elliptic $\left(\mathrm{v}_{2}\right)$ and triangular $\left(v_{3}\right)$ flow coefficients measured for charged hadrons in these three systems [10]. Only $0-5 \%$ most central collisions were used for these measurements. One can see that values of $v_{2}$ and $v_{3}$ follow exactly the same hierarchy as the mean second and third order spatial eccentricities. The elliptic flow $v_{2}$ is about the same in $\mathrm{d}+\mathrm{Au}$ and ${ }^{3} \mathrm{He}+\mathrm{Au}$ collisions and it is significantly smaller in $\mathrm{p}+\mathrm{Au}$ collisions. The triangular flow is the largest in ${ }^{3} \mathrm{He}+\mathrm{Au}$ collisions and it is about the same in $\mathrm{d}+\mathrm{Au}$ and $\mathrm{p}+\mathrm{Au}$ collisions. The obvious conclusion is that there is a direct connection between the initial nuclear overlap geometry and the final state particle momentum anisotropy described with $v_{2}$ and $v_{3}$ coefficients.
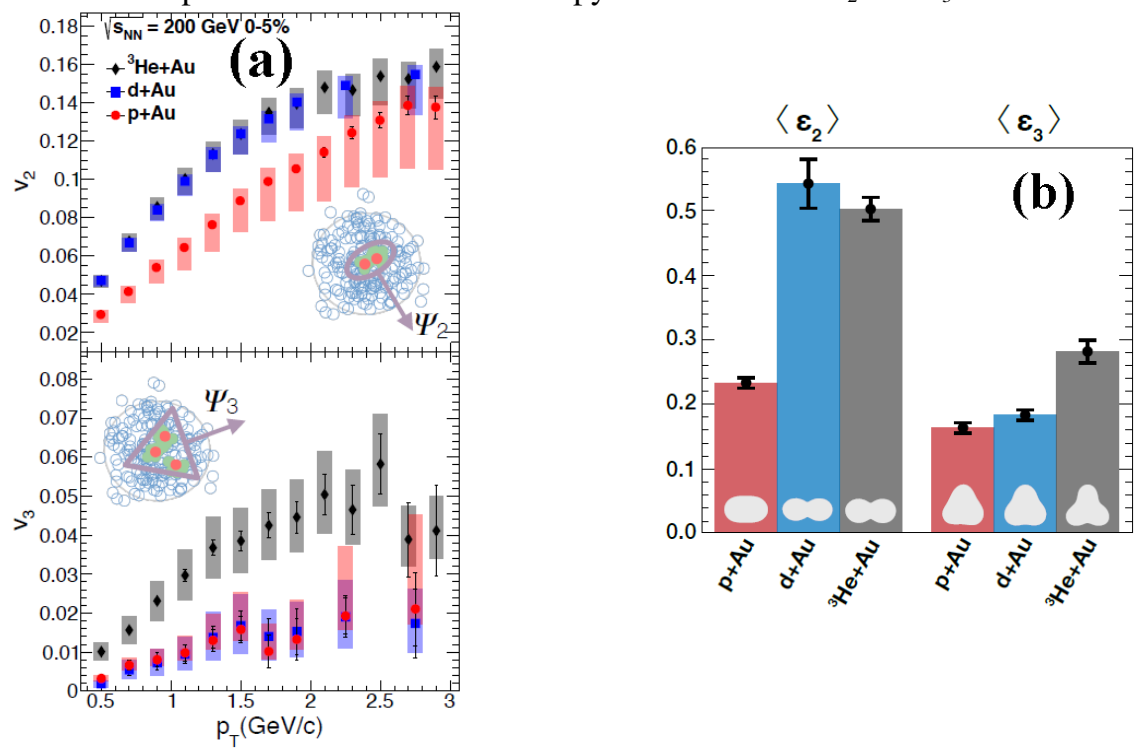

Figure 4. (a) Elliptic $\left(\mathrm{v}_{2}\right)$ and triangular $\left(\mathrm{v}_{3}\right)$ flow measured for charged hadrons in 0-5\% most central $\mathrm{p} / \mathrm{d} /{ }^{3} \mathrm{He}+\mathrm{Au}$ collisions at $\sqrt{ }_{\mathrm{NN}}=200 \mathrm{GeV}$. (b) Mean values of the second and third order spatial eccentricities calculated using the Glauber Monte-Carlo model in 0-5\% most central $\mathrm{p} / \mathrm{d} /{ }^{3} \mathrm{He}+\mathrm{Au}$ collisions at $\sqrt{\mathrm{s}_{\mathrm{NN}}}=200 \mathrm{GeV}$ 
In order to understand the mechanism of transformation, in Fig. 5, we compare the measured values of $v_{2}$ and $v_{3}$ to different model calculations. The blue and red curves correspond to two different hydrodynamic model calculations [11,12]. One can see that both models reasonably well describe the results for $v_{2}$ and $v_{3}$ simultaneously in three different collision systems. These models also describe the particle production spectra. The success of hydrodynamic models serves as a strong evidence in favour of quark-gluon plasma droplets formation in high-multiplicity collisions of small systems. The measured results can also be compared to predictions of the AMPT event generator [13]. The AMPT is a microscopic model that uses the Glauber initial conditions, string melting to partons, partonic and hadronic cascades and parton coalescence as a hadronization mechanism. In the AMPT, the translation of the initial spatial anisotropy to the final state azimuthal momentum anisotropy occurs via parton-parton scattering with a modest interaction cross section. The AMPT provides a decent description of the $v_{2}$ and $v_{3}$ coefficients in all three systems but only at very low momentum $\mathrm{p}_{\mathrm{T}}<1-1.5 \mathrm{GeV} / \mathrm{c}$. The main problem of the AMPT is that it cannot describe the flow simultaneously in small and large collision systems with a consistent set of model parameters. Another class of models explains the final-state momentum anisotropies via initial-state colour correlations computed in the Colour Glass Condensate effective field theory. The calculations of one of such models are shown by green curves in the figure [14]. The model reasonably well describes the elliptic flows in three systems. It also described the $\mathrm{v}_{3}$ results in ${ }^{3} \mathrm{He}+\mathrm{Au}$ collisions. However, it significantly overestimates $\mathrm{v}_{3}$ in $\mathrm{p}+\mathrm{Au}$ and $\mathrm{d}+\mathrm{Au}$ collisions.

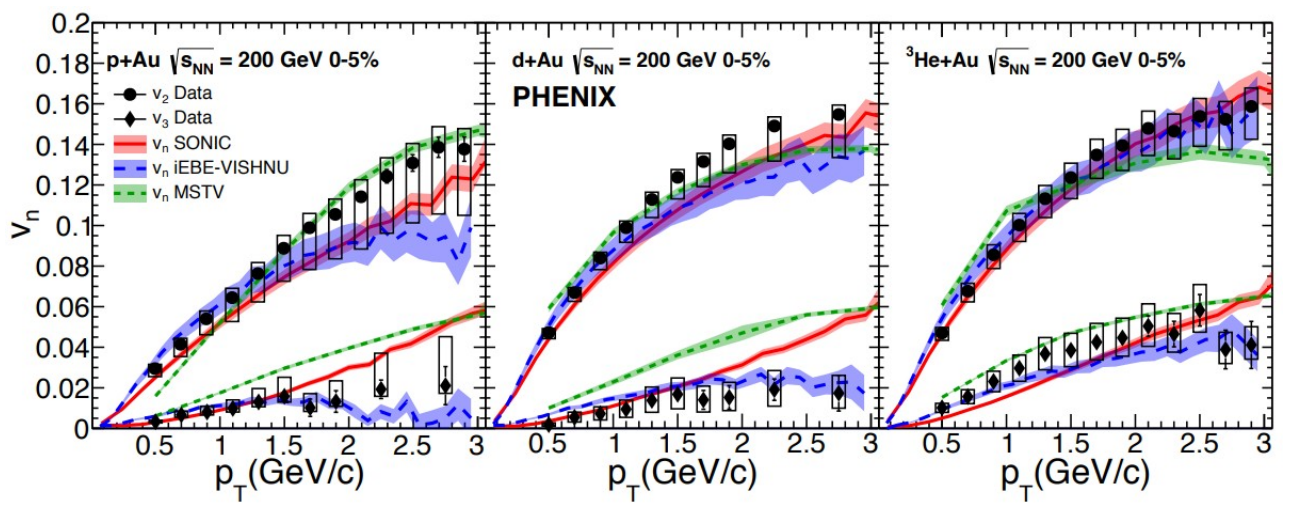

Figure 5. Elliptic $\left(\mathrm{v}_{2}\right)$ and triangular $\left(\mathrm{v}_{3}\right)$ flows measured for charged hadrons in $0-5 \%$ most central $\mathrm{p} / \mathrm{d} /{ }^{3} \mathrm{He}+\mathrm{Au}$ collisions at $\sqrt{\mathrm{s}_{\mathrm{NN}}}=200 \mathrm{GeV}$ compared to different model calculations

\subsection{Energy scan program}

The purpose of the energy scan program was to understand how the particle flow depends on a collision energy. Fig. 6 shows the elliptic flow measured for charged hadrons in $0-5 \%$ most central $d+A u$ collisions at $\sqrt{ }_{\mathrm{N}_{\mathrm{N}}}=200,62.4,39$ and $19.6 \mathrm{GeV}$. Please note that these 
results are not corrected for non-flow contributions, neither are they accounted in systematic uncertainties [15]. Hydrodynamic models reasonably well reproduce data at $\sqrt{\mathrm{s}_{\mathrm{NN}}}$

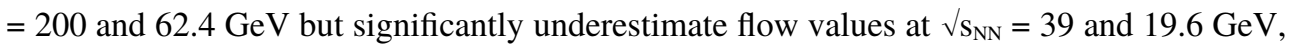
especially at higher transverse momenta $[11,12,16]$. In the figure, one can see the data compared to the AMPT calculations [13]. The blue curves correspond to the case when the elliptic flow is calculated in the same way as in the data, via the angular distribution of the final-state hadrons. Such estimations naturally include non-flow contributions, just like the analysis of real data. The green curves were obtained using the parton event plane and these curves should be much less sensitive to non-flow contributions and present the true flow. One can see that according to the AMPT, the non-flow contributions become more important at lower collision energies and higher transverse momenta. According to the AMPT, a strong collective flow signal is observed even at the lowest collision energy of $\sqrt{ }_{\mathrm{NN}}=19.6 \mathrm{GeV}$ and the signal is inconsistent with non-flow contributions only.

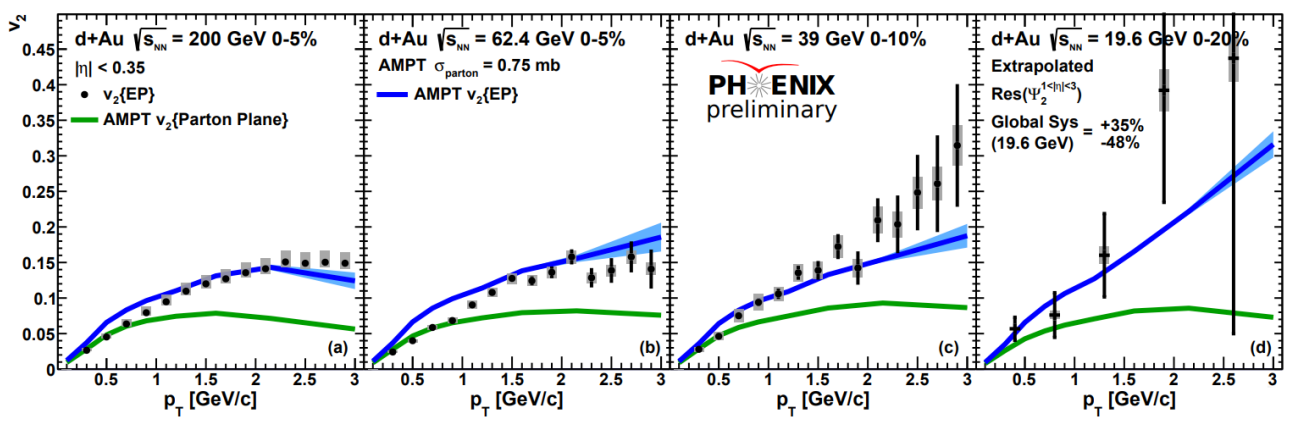

Figure 6. Elliptic $\left(\mathrm{v}_{2}\right)$ flow measured for charged hadrons in $0-5 \%$ most central $\mathrm{d}+\mathrm{Au}$ collisions at $\sqrt{ }_{\mathrm{S}_{\mathrm{NN}}}=200,62.4,39$ and $19.6 \mathrm{GeV}$ compared the AMPT model calculations

\section{Conclusions}

The PHENIX collaboration is still active, it continues to analyse data and delivers results of great importance for the high-energy physics.

PHENIX observed the excessive production of low- $\mathrm{p}_{\mathrm{T}}$ direct photons in most central $\mathrm{p}+\mathrm{Au}$ collisions at ${\sqrt{\mathrm{s}_{\mathrm{NN}}}}=200 \mathrm{GeV}$. This is a first observation of the thermal photon production in collisions of small systems at RHIC consistent with formation of the QGP droplets in such collisions. The observation of the universal scaling of thermal photon yields divided by the charged particle multiplicity density to the power of 1.25 in heavy-ion collisions at different centralities and energies in the range $\sqrt{ }_{\mathrm{S}_{\mathrm{NN}}}=39-2760 \mathrm{GeV}$ needs a solid theoretical explanation. The scaling may indicate the large direct photon production near the phase transition to the hadronic phase.

The final-state particle momentum anisotropy observed in high-multiplicity collisions of small systems at RHIC is a result of the initial spatial anisotropy of the nuclear overlap region. The mechanism of such transformation is not fully constrained. At the moment, the whole set of measurements at the energy of $\sqrt{ }_{\mathrm{NN}}=200 \mathrm{GeV}$ is best and uniquely described 
by hydrodynamic model calculations, which suggest the formation of QGP droplets in such collisions. The energy scan program shows the evidence of the collective flow in most central $\mathrm{d}+\mathrm{Au}$ collisions even at the lowest energy of $\sqrt{ }_{\mathrm{NN}}=19.6 \mathrm{GeV}$. The interpretation of these results is complicated by significant non-flow contributions.

Acknowledgement. This work was partially supported by the National Research Nuclear University $\mathrm{MEPhI}$ in the framework of the Russian Academic Excellence Project (contract No. 02.a03.21.0005, 27.08.2013).

\section{References}

1. K. Adcox et al., Nucl. Phys. A 757, 184-283 (2005)

2. A. Adare et al., Phys. Rev. C 91(6), 064904 (2015)

3. A. Adare et al., Phys. Rev. C 94(6), 064901 (2016)

4. J. Adam et al., Phys. Lett. B 754, 235-248 (2016)

5. A. Adare et al., arXiv:1805.04066 [hep-ex] (2018)

6. A. Adare et al., arXiv:1805.04084 [hep-ex] (2018)

7. A. Adare et al., Phys. Rev. C 87, 054907 (2013)

8. D. Abelev et al., Phys. Lett. B 719, 29-41 (2013)

9. S. Chatrchyan et al., Phys. Lett. B 718, 795-814 (2013)

10. A. Adare et al., Phys. Rev. C 97(6), 064904 (2016)

11. M. Habich et al., Eur. Phys. J. C 75, 15 (2015)

12. C. Shen et al., Phys. Rev. C 95, 014906 (2017)

13. Z.-W. Lin et al., Phys. Rev. C 72, 064901 (2005)

14. M. Mace et al., Phys. Rev. Lett. 121, 052301 (2018)

15. C. Aidala et al., Phys. Rev. C 96(6), 064905 (2017)

16. P. Romatschke et al., Eur. Phys. J. C 75, 305 (2015) 